AB0631 THE PREVALENCE AND FACTORS ASSOCIATED WITH CORONARY HEART DISEASE IN PATIENTS WITH GOUT

E. Markelova ${ }^{1}$, M. Eliseev ${ }^{2}$, E. llinykh ${ }^{3}$, S. Glukhova ${ }^{4}$, T. Popkova5. ${ }^{1}$ V.A. NASONOVA RESEARCH INSTITUTE OF RHEUMATOLOGY, Laboratory of Systemic Rheumatic Diseases, Moscow, Russian Federation; ${ }^{2}$ V.A. Nasonova Research Institute of Rheumatology, Moscow, Laboratory of Crystal Diseases, Moscow, Russian Federation; ${ }^{3}$ V.A. NASONOVA RESEARCH INSTITUTE OF RHEUMATOLOGY, Laboratory of Crystal Diseases, Moscow, Russian Federation; ${ }^{4}$ Moscow, Laboratory of Systemic Rheumatic Diseases, Moscow, Russian Federation; ${ }^{1}$ V.A. NASONOVA RESEARCH INSTITUTE OF RHEUMATOLOGY, Laboratory of Systemic Rheumatic Diseases, Moscow, Russian Federation

Background: Gout is associated with increased risk of cardiovascular disease (CVD) morbidity and mortality. Therefore, an association between coronary heart disease (CHD) and gout deserves careful examination.

Objectives: The aim of this study was to determine the prevalence of $\mathrm{CHD}$ and factors associated with $\mathrm{CHD}$ in patients (pts) with gout.

Methods: 286 male patients fulfilling Wallace proposed criteria for gout were included: age 51.2 [42.8;59.4] years (ys), disease duration - 6.2 [3.8;12.1] ys. All patients underwent standard clinical examination, screening traditional risk factors (TRF) of CVD, blood chemistry test with estimation of serum uric acid, serum creatinine, C-reactive protein (CRP), as well as lipid profile. Carotid intima-media thickness (CIMT) was measured using a high-resolution B-mode ultrasound machine. CHD included history of angina pectoris and/or myocardial infarction. We estimated the adjusted odds ratio (OR) and 95\% confidence interval (95\% $\mathrm{Cl})$.

Results: CHD was found in 111 out of the 286 pts (38.8\%). Compared to individuals with CHD, participants without CHD were older (56.7[52.1; 61.1] vs 46.2[40.6; $53.4] \mathrm{ys})$, had longer duration of gout $(9.3[4.7 ; 15.1]$ vs $5.6[3.3 ; 9.7]$ ys), higher number of joints involved during disease course $(8[6 ; 15]$ vs $6[4 ; 10])$, duration of smoking $(24[10 ; 40]$ vs 20[10; 28]), higher serum creatinine level accordingly, (for all $p<0.05)$. The frequency of family history of $\mathrm{CHD}(63 \%$ vs $46.8 \%)$, intraosseous tophi $(61.3 \%$ vs $33.1 \%)$, was higher in pts with $\mathrm{CHD}$ compared pts without $\mathrm{CHD}$ accordingly, (for all $p<0.01$ ). Prevalence of arterial hypertension, diabetes mellitus, nephrolithiasis, heart failure and renal failure was greater in pts with $\mathrm{CHD}$ than pts without $\mathrm{CHD}(\mathrm{p}<0.001)$. Gout pts with $\mathrm{CHD}$ had a significantly higher clMT compare to those without CHD - 0.95[0.8;1.08]/0.8[0.7;0.9] accordingly, $\mathrm{p}<0.001$. We didn't find differences of lipid profile, serum uric acid, and CRP level in gout pts with/without $\mathrm{CHD}$.

Abdominal obesity (OR, 5.5; 95\% Cl, 2.2-13.6), body mass index $>30 \mathrm{~kg} / \mathrm{m}^{2}(\mathrm{OR}$, $5.8 ; 95 \% \mathrm{Cl}, 1.8-18.5)$, family history of $\mathrm{CHD}(\mathrm{OR}, 2.7 ; 95 \% \mathrm{Cl}, 1.3-5.4)$, disease duration of gout more 10 ys $(\mathrm{OR}, 2.6 ; 95 \% \mathrm{Cl}, 1.3-5.1)$, age of gout onset $<35$ ys (OR, 3.0; 95\% Cl, 1.5-6.1), intraosseous tophi (OR, 3.1; 95\% Cl, 1.4-7.0), C-RP $(\mathrm{OR}, 2.2 ; 95 \% \mathrm{Cl}, 1.1-4.7)$, renal failure $(\mathrm{OR}, 18,8 ; 95 \% \mathrm{Cl}, 1.1-312.9)$, increased the risk for CHD in patients with a gout.

Conclusion: The prevalence of CHD was $38.8 \%$ among individuals with gout. Our study showed that both TRFs of CVD and the severity of gout and a history of renal failure contribute to the development of $\mathrm{CHD}$ in patients with gout.

Disclosure of Interests: None declared.

DOI: 10.1136/annrheumdis-2021-eular.1241

\section{AB0632 COMORBIDITIES AND DRUG-INDUCED LIVER INJURY DUE TO NSAIDS INTAKE IN PATIENTS WITH GOUT}

E. Mikhnevich ${ }^{1}$, P. Tatsiana ${ }^{1}$, R. Tatsiana ${ }^{1}$, H. Diana ${ }^{1} .{ }^{1}$ Belarusian State Medical University, Internal Disease, Minsk, Belarus

Background: One of the side effects of NSAIDs is hepatotoxicity or Drug-Induced Liver Injury (DILI). Despite the short course of NSAIDs, the patients with gout may develop DILI. At the same time, besides NSAIDs, there are additional factors that affect the functional state of the liver: demography, comorbidities or concomitant medication (1).

Objectives: To determine comorbidities associated with the risk of DILI when taking NSAIDs during the attack of gouty arthritis.

Methods: Our retrospective study included 200 patients with GA. Inclusion criteria for the study: presence of gout (ACR, 1977), initially normal serum ALT level, NSAIDs as anti-inflammatory drugs in gout. Exclusion criteria: presence of a known liver disease. All patients were divided into 2 groups: those who developed DILI $(n=86)$ and the control group without cytolysis during NSAID treatment $(n=114)$. The severity of the cytolytic syndrome was revealed as follows: mild form (2-3-fold excess of the ALT norm) in 81,4\% ( $n=70)$; moderate (3-5 times higher ALT) in $15.1 \%(n=13)$; severe $(5-10$ fold excess of ALT) in $3.4 \%$ $(n=3)$. The mean age of $55(49-60)$ years and $54(44-59.5)$ years as well as the sex distribution (men made $90.1 \%$ and $93.3 \%$, respectively) were compatible in both groups $(p>0,05)$. When studying the age distribution, statistical differences were revealed in the groups under 52 years $\left(p^{<} 0,05\right)$, so, this parameter was included into logistic regression model. During the follow-up period, all patients took NSAIDs above average doses. Patients in both groups were comparable in duration of gout. The following parameters were compared: AH, IHD, diabetes mellitus, obesity, dyslipidemia, metabolic syndrome according to ATPIII, CKD, alcohol abuse, age under 52 .

Results: After conducting logistic regression analysis, the next comorbidities have been revealed to be statistically significant: obesity (OR $-2,15 ; 95 \% \mathrm{Cl}$ 1,16-3,98), dyslipidemia (OR - 3,41; 95\% Cl 1,56-7,44) and alcohol abuse (OR $-2,45 ; 95 \% \mathrm{Cl} 1,22-4,91)$. Worth to note that age under 52 was also a risk factor for DILI (OR - 2,14; 95\%Cl 1,13-4,04). Thus, among our patients, the younger ones with an unhealthy lifestyle and no serious comorbidities, including cardiovascular, were at a greater risk for developing DILI while taking NSAIDs during the gout attack.

Conclusion: In the study group of patients with gout, the hepatotoxicity (cytolysis syndrome) risk due to NSAIDs intake increased in younger patients in the presence of signs of metabolic syndrome (obesity, dyslipidemia) and alcohol abuse. REFERENCES:

[1] European Association for the Study of the Liver. EASL Clinical Practice Guidelines Drug-induced liver injury. J. of Hepatology. 2019; 30: 1-40.

Disclosure of Interests: None declared.

DOI: 10.1136/annrheumdis-2021-eular.1381

\section{AB0633 HEALTH RELATED QUALITY OF LIFE IN GOUT, PSORIATIC ARTHRITIS, RHEUMATOID ARTHRITIS AND ANKYLOSING SPONDYLITIS: RESULTS FROM A QUESTIONNAIRE STUDY}

A. Landgren ${ }^{1}$, M. Dehlin ${ }^{1}$, L. T. H. Jacobsson ${ }^{1}$, U. Bergsten ${ }^{2}$, E. Klingberg ${ }^{1}$ ${ }^{1}$ Institute of Medicine, Department of Rheumatology and Inflammation Research, Sahlgrenska Academy, University of Gothenburg, Gothenburg, Sweden; ${ }^{2}$ County of Halland, R\&D Department at Region Halland, Halmstad, Halmstad, Sweden

Background: Gout, psoriatic arthritis (PsA), rheumatoid arthritis (RA), ankylosing spondylitis (AS), are common inflammatory joint diseases (IJD) that substantially affect HRQoL (Health Related Quality of Life). To our knowledge, this is the first study comparing HRQoL in gout, PsA, RA and AS in the same setting.

Objectives: To compare HRQoL assessed with the Short Form health survey (SF-36) between gout, PsA, RA and AS.

Methods: We performed a cross-sectional questionnaire study in Sweden. Individuals $\geq 18$ years and with at least one ICD-10 diagnosis for gout, PsA, RA, or AS, recorded at a health care visit to a physician at a rheumatology clinic (for all diagnoses) or at a primary care center (for gout patients) from 2015 through 2017 were identified. Patients with gout $(n=1589), \operatorname{PsA}(n=1200), R A(n=1246)$ and $A S(n=1095)$ were sent a questionnaire including questions regarding Visual Analogue Scales (VAS) for General Health, Pain, Fatigue, Health Asssessment Questionnaire (HAQ), as well as the SF-36. The eight domains (a higher score indicating a better health status) as well as the overall physical (PCS) and mental (MCS) component scores (a value of 50 corresponds to unaffected HRQoL) of the SF-36 are presented. Respondents were matched on age, sex. ANOVA was used for between group comparisons of mean values. For non-normally distributed data, Kruskal Wallis test was used. For comparing distributions, the Chi-Square test was used.

Results: In total, 2896 (56.5\%) individuals responded. After matching for age, sex, 249 individuals per diagnosis group remained. Gout patients reported significantly higher scores on SF-36 subscales as well as PCS (consistent with results seen before matching for age and sex). Lower values of HRQoL were noted in physical domains as compared to mental domains in all IJDs. Disability measured by HAQ was significantly lower in gout and VAS scores were more favourable compared to other IJDs.

Conclusion: Patients with gout report higher HRQOL compared to PsA, RA, AS 
Table 1. Age-matched comparisons across diagnoses

\begin{tabular}{|c|c|c|c|c|c|c|c|c|c|c|c|}
\hline & Gout & PsA & RA & AS & $\begin{array}{l}p- \\
\text { value }\end{array}$ & $\begin{array}{l}\mathrm{p} \text {-value } \\
\text { Gout }\end{array}$ & $\begin{array}{l}\text { ep-value } \\
\text { Gout }\end{array}$ & $\begin{array}{l}\text { p-val } \\
\text { Gout }\end{array}$ & $\begin{array}{l}\text { p-val } \\
\text { s PsA }\end{array}$ & $\begin{array}{l}\text { ep-va } \\
\text { s PsA }\end{array}$ & $\begin{array}{l}\text { ep-value } \\
\text { s RA v }\end{array}$ \\
\hline & & & & & & vs PsA & vs RA & AS & $\mathrm{RA}$ & AS & AS \\
\hline Total (n) & 249 & 249 & 249 & 249 & $\mathrm{~N} / \mathrm{A}$ & & & & & & \\
\hline $\begin{array}{l}\text { Age, mean } \\
\text { (SD) }\end{array}$ & $\begin{array}{l}62.5 \\
(11.2)\end{array}$ & $\begin{array}{l}64.2 \\
(10.2)\end{array}$ & $\begin{array}{l}63.1 \\
(11.6)\end{array}$ & $\begin{array}{l}62.8 \\
(10.5)\end{array}$ & NS & & & & & & \\
\hline $\begin{array}{l}\text { Education } \\
\leq 12 \text { years, } \\
\mathrm{n}(\%)\end{array}$ & $\begin{array}{l}132 \\
(53.0)\end{array}$ & $\begin{array}{l}148 \\
(59.4)\end{array}$ & $\begin{array}{l}160 \\
(64.3)\end{array}$ & $\begin{array}{l}139 \\
(55.8)\end{array}$ & NS & & & & & & \\
\hline $\begin{array}{l}\mathrm{HAQ} \text {, mean } \\
\text { (SD) }\end{array}$ & $\begin{array}{l}0.19 \\
(0.40)\end{array}$ & $\begin{array}{l}0.48 \\
(0.55)\end{array}$ & $\begin{array}{l}0.60 \\
(0.63)\end{array}$ & $\begin{array}{l}0.58 \\
(0.58)\end{array}$ & *** & 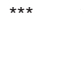 & $\star * \star *$ & $\star \star \star *$ & NS & NS & NS \\
\hline $\begin{array}{l}\text { VAS General } \\
\text { Health, } \\
\text { mean (SD) }\end{array}$ & $\begin{array}{l}2.6 \\
(2.3)\end{array}$ & $\begin{array}{l}3.7 \\
(2.4)\end{array}$ & $\begin{array}{l}3.8 \\
(2.6)\end{array}$ & $\begin{array}{l}4.0 \\
(2.3)\end{array}$ & *** & 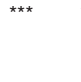 & 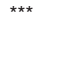 & 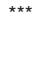 & NS & NS & NS \\
\hline $\begin{array}{l}\text { VAS Pain, } \\
\text { mean (SD) }\end{array}$ & $\begin{array}{l}2.6 \\
(2.4)\end{array}$ & $\begin{array}{l}4.0 \\
(2.6)\end{array}$ & $\begin{array}{l}3.8 \\
(2.6)\end{array}$ & $\begin{array}{l}4.2 \\
(2.4)\end{array}$ & 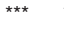 & $* \star \star$ & 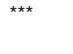 & 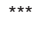 & NS & NS & NS \\
\hline $\begin{array}{l}\text { VAS Fatigue, } \\
\text { mean (SD) }\end{array}$ & $\begin{array}{l}3.6 \\
(2.6)\end{array}$ & $\begin{array}{l}4.5 \\
(2.7)\end{array}$ & $\begin{array}{l}4.3 \\
(2.6)\end{array}$ & $\begin{array}{l}4.9 \\
(2.5)\end{array}$ & 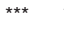 & $* * \star$ & * & $\star \star \star *$ & NS & NS & * \\
\hline \multicolumn{12}{|c|}{$\begin{array}{l}\text { SF-36 } \\
\text { domains, } \\
\text { median (IQR) }\end{array}$} \\
\hline $\begin{array}{l}\text { Physical } \\
\text { function }\end{array}$ & $\begin{array}{l}90.0 \\
(75.0- \\
95.0)\end{array}$ & $\begin{array}{l}70.0 \\
-(51.3- \\
95.0)\end{array}$ & $\begin{array}{l}70.0 \\
(45.0- \\
90.0)\end{array}$ & $\begin{array}{l}75.0 * \\
-(50.0- \\
90.0)\end{array}$ & *** & 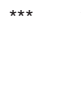 & 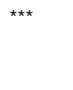 & $\star \star \star$ & NS & NS & NS \\
\hline Role physical & $\begin{array}{l}100.0 \\
(33.3- \\
100.0)\end{array}$ & $\begin{array}{l}50.0 \\
-(0.0- \\
100.0)\end{array}$ & $\begin{array}{l}50.0 \\
(0.0- \\
100.0)\end{array}$ & $\begin{array}{l}50.0 \\
(0.0- \\
100.0)\end{array}$ & *** & 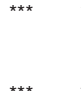 & 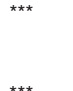 & $\star \star \star$ & NS & NS & NS \\
\hline Bodily pain & $\begin{array}{l}72.0 \\
(42.0- \\
100.0)\end{array}$ & $\begin{array}{r}52.0 \\
-(41.0- \\
74.0)\end{array}$ & $\begin{array}{l}52.0 \\
(41.0- \\
74.0)\end{array}$ & $\begin{array}{l}52.0 * \\
-(41.0- \\
64.0)\end{array}$ & *** & 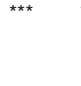 & 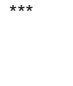 & 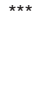 & NS & NS & NS \\
\hline $\begin{array}{l}\text { General } \\
\text { Health }\end{array}$ & $\begin{array}{l}67.0 \\
(47.8- \\
80.8)\end{array}$ & $\begin{array}{l}56.0 \\
(35.0- \\
72.0)\end{array}$ & $\begin{array}{l}55.0 \\
-(37.0- \\
72.0)\end{array}$ & $\begin{array}{l}50.0 * \\
(35.0- \\
71.0)\end{array}$ & *** & 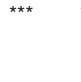 & 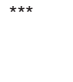 & 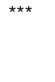 & NS & NS & NS \\
\hline Vitality & $\begin{array}{l}65.0 \\
(50.0- \\
77.5)\end{array}$ & $\begin{array}{l}57.5 \\
-(37.5- \\
75.0)\end{array}$ & $\begin{array}{l}57.5 \\
(42.5- \\
72.5)\end{array}$ & $\begin{array}{l}50.0 * \\
-(32.5- \\
70.0)\end{array}$ & *** & * & * & 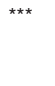 & NS & NS & NS \\
\hline $\begin{array}{l}\text { Social } \\
\quad \text { function }\end{array}$ & $\begin{array}{l}100.0 \\
(68.8- \\
100.0)\end{array}$ & $\begin{array}{l}81.3 \\
-(57.8- \\
100.0)\end{array}$ & $\begin{array}{l}81.3 \\
(67.2- \\
100.0)\end{array}$ & $\begin{array}{l}81.3 \text { * } \\
(50.0- \\
100.0)\end{array}$ & *** & $\star *$ & $\star \star$ & $\star \star \star$ & NS & NS & NS \\
\hline $\begin{array}{l}\text { Role } \\
\text { emotional }\end{array}$ & $\begin{array}{l}100.0 \\
(66.7- \\
100.0)\end{array}$ & $\begin{array}{l}100.0 \\
(33.3- \\
100.0)\end{array}$ & $\begin{array}{r}100.0 \\
-(33.3- \\
100.0)\end{array}$ & $\begin{array}{l}100.0 \text { * } \\
-(33.3- \\
100.0)\end{array}$ & & NS & $\star \star$ & $\star \star$ & NS & NS & NS \\
\hline Mental health & $\begin{array}{l}82.0 \\
(66.0- \\
92.0)\end{array}$ & $\begin{array}{l}78.0 \\
-(60.0- \\
92.0)\end{array}$ & $\begin{array}{l}78.0 \\
(60.0- \\
92.0)\end{array}$ & $\begin{array}{l}73.0 \text { * } \\
-(60.0- \\
88.0)\end{array}$ & & NS & * & 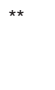 & NS & NS & NS \\
\hline \multicolumn{12}{|c|}{$\begin{array}{l}\text { SF-36 } \\
\text { summary } \\
\text { scores, } \\
\text { median (IQR) }\end{array}$} \\
\hline $\begin{array}{l}\text { Physical } \\
\text { component }\end{array}$ & $\begin{array}{l}48.4 \\
t(38.8-\end{array}$ & $\begin{array}{l}40.2 \\
-(28.5-\end{array}$ & $\begin{array}{l}40.2 \\
-(28.2-\end{array}$ & $\begin{aligned} & 38.7 \text { * } \\
- & (30.1-\end{aligned}$ & *** & 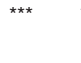 & 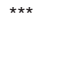 & 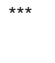 & NS & NS & NS \\
\hline $\begin{array}{l}\text { score } \\
\text { Mental }\end{array}$ & $\begin{array}{l}54.7) \\
51.7\end{array}$ & $\begin{array}{l}50.2) \\
51.0\end{array}$ & $\begin{array}{l}49.0) \\
50.7\end{array}$ & $\begin{array}{l}48.2) \\
47.7\end{array}$ * & * & NS & NS & * & NS & NS & NS \\
\hline $\begin{array}{l}\text { component } \\
\text { score }\end{array}$ & $\begin{array}{c}t(43.7- \\
56.5)\end{array}$ & $\begin{array}{l}(39.2- \\
56.4)\end{array}$ & $\begin{array}{l}-(39.6- \\
56.3)\end{array}$ & $\begin{array}{l}-(37.1- \\
55.3)\end{array}$ & & & & & & & \\
\hline
\end{tabular}

$\mathrm{HAQ}=$ Health Assessment questionnairel $\mathrm{RQ}=$ Interquartile range $\mathrm{SD}=\mathrm{Standard}$ deviationVAS $=$ Visual Analogue ScaleNS $=$ non-significant ${ }^{*}=$ significant at $\mathrm{p}<0.05^{* *}=$ significant at $\mathrm{p}<0.01^{\star \star \star}=$ significant at $\mathrm{p}<0.001$

Disclosure of Interests: None declared.

DOI: 10.1136/annrheumdis-2021-eular.1582

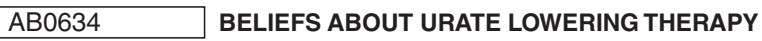 DIFFER BETWEEN GENERAL PRACTITIONERS AND RHEUMATOLOGISTS}

F. Veenstra $^{1}$, J. Vriezekolk' ${ }^{1}$ H. J. Schers ${ }^{2}$, N. Van Herwaarden ${ }^{1,3}$, B. Van den Bemt ${ }^{4,5}$, M. Flendrie'. ' Sint Maartenskliniek, Rheumatology, Ubbergen, Netherlands; ${ }^{2}$ Radboud University Medical Center, Primary and Community Care, Nijmegen, Netherlands; ${ }^{3}$ Radboud University Medical Center, Rheumatology, Nijmegen, Netherlands; ${ }^{4}$ Sint Maartenskliniek, Rheumatology \& Pharmacy, Ubbergen, Netherlands; ${ }^{5}$ Radboud University Medical Center, Pharmacy, Nijmegen, Netherlands

Background: Uric acid lowering therapy (ULT) can be effective in gout if taken correctly (1), but non-adherence is a known problem (2). Although patients' adherence barriers regarding ULT, such as lack of knowledge on disease and therapy, and beliefs, have been widely studied (3), less is known physicians' beliefs.

Objectives: To investigate the physicians' beliefs on ULT treatment in both primary and secondary care in the Netherlands.

Methods: Cross-sectional study among all rheumatologists of the Sint Maartenskliniek and to all General Practitioners (GPs) of 17 practices, participating in the practice-based research network Family Medicine Network Nijmegen,
Netherlands. All participants filled out the beliefs about medication questionnaire (BMQ), adjusted for physicians; in addition data were collected on physician characteristics including working experience in total and gout consultations. The BMQ consists of two parts, a specific part with 10 questions about beliefs on necessity $(\mathrm{N}=5)$ and concerns $(\mathrm{N}=5)$ regarding ULT and a general part with 8 questions about beliefs on harms $(\mathrm{N}=4)$ and overuse $(\mathrm{N}=4)$ regarding medication in general Each question is scored on a Likert-scale from 1-5, a higher score corresponds to higher beliefs on that category. Sum scores for all four categories were calculated. With the total scores on the specific $B M Q$ including necessity and concerns beliefs, physicians were classified in four different categories (Table 1)(4).

Results: In total 112 physicians received the questionnaire, 28 of 37 rheumatologists $(76 \%)$ and 45 of 75 GPs $(60 \%)$ responded. Rheumatologists had less years of experience than GPs (median 8.5 years (IQR $3-14$ ) versus (vs) 13 years (IQR 8-20)) and less hours of patient contact per week (15 hours (IQR $8.5-20$ ) vs 24 hours (IQR $20-30)$ ). Rheumatologists reported more consultations for gout per week than GPs: median 4 (IQR 1 - 6.6) vs 1 (IQR 0.2-1).

Rheumatologists scored higher on the BMQ necessity scale, 17.5 (95\% Cl 16.6 - 18.5) compared to GPs 16.1 (95\% Cl 15.1 - 17.1). GPs scored higher on BMQ concern scale, $12.1(95 \% \mathrm{Cl} 11.4-12.7)$ compared to rheumatologists, 10.4 (95\% Cl 9.7 - 11.2). On medication in general, GPs scored higher on both overuse and harms scales compared to rheumatologists, $11.3(95 \% \mathrm{Cl} 10.7-$ 12) versus $9.9(95 \% \mathrm{Cl} 9-10.7)$ and $8.3(95 \% \mathrm{Cl} 7.9-8.7)$ vs $7.1(95 \% \mathrm{Cl} 6.5$ $-7.7)$, respectively. Table 1 shows classification according to the BMQ specific for both groups.

Table 1. Classification based on the adapted BMQ

\begin{tabular}{lll}
\hline & Rheumatologists (N=28) & $\begin{array}{l}\text { GPs } \\
(\mathrm{N}=45)\end{array}$ \\
\hline Acceptant (\%) & $19(67.9)$ & $16(35.6)$ \\
Ambivalent (\%) & 0 & $8(17.8)$ \\
Sceptic (\%) & $1(3.6)$ & $5(11.1)$ \\
Indifferent (\%) & $8(28.6)$ & $16(35.6)$ \\
\hline
\end{tabular}

Conclusion: For the majority of rheumatologist the necessity beliefs outweighs concern beliefs towards the use of ULT outweighs concern beliefs. GPs show a more heterogenic profile, including acceptant, indifferent and ambivalent beliefs towards ULT use. GPs also show higher harm and overuse concerns regarding medication in general. Next step is to investigate possible associations with actual ULT use and disease outcome.

REFERENCES:

[1] Doherty M, et al. Lancet (London, England). 2018;392(10156):1403-12.

[2] Reach G. Joint, bone, spine: revue du rhumatisme. 2011;78(5):456-9.

[3] Harrold LR et al. Chronic illness. 2010;6(4):263-71.

[4] Toelichting BMQ. [updated 2011-12-10; cited 2021-01-28] Available trough: https://meetinstrumentenzorg.nl/instrumenten/beliefs-about-medicine -questionnaire-bmq-bmq-specific-bmq-general/

Acknowledgements: This study is sponsored by Grünenthal

Disclosure of Interests: Frouwke Veenstra: None declared., Johanna Vriezekolk: None declared., Henk J Schers: None declared., Noortje van Herwaarden: None declared., Bart van den Bemt Speakers bureau: Pfizer, AbbVie, UCB, Biogen and Sandoz, Consultant of: Delivered consultancy work for UCB, Novartis and Pfizer, Grant/research support from: UCB, Pfizer and Abbvie, Marcel Flendrie Consultant of: M. Flendrie has received consultancy fees from Menarini and Grunenthal., Grant/ research support from: M. Flendrie has received grants from Menarini and Grunenthal. DOI: 10.1136/annrheumdis-2021-eular.1834

\section{AB0635 INDEX BY CAROTID DOPPLER IN PATIENTS WITH MONOSODIUM URATE DEPOSITION ARTHRITIS OF THE HOSPITAL DOCENTE PADRE BILLINI, DOMINICAN REPUBLIC}

A. Cornelio ${ }^{1,2}$, J. Santana Peralta ${ }^{2}$, T. Polanco Mora ${ }^{2}$, Y. Cruz ${ }^{2}$, E. Rodriguez Bautista $^{2}$, T. Valdez ${ }^{2}$, R. Muñoz ${ }^{2}$, A. Feriz ${ }^{2}{ }^{1}$ Hospital Docente Padre Billini, Reumatologia, Santo Domingo, Dominican Republic; ${ }^{1}$ Hospital Docente Padre Billini, Reumatologia, Santo Domingo, Dominican Republic

Background: Hyperuricemia and gout are risk factors for atherosclerosis subclinical and cardiovascular complications. (1) However, it is still debated whether uric acid is an independent predictor of cardiovascular risk. (2) Vascular Doppler and in particular the measurement of the intima-media thickness and the detection of plaques are useful in the evaluation of cerebrovascular disease and cardiovascular risk. (3) An intima-media thickness greater than $0.9 \mathrm{~mm}$ and / or the presence of atheromatous plaques in the carotid are predictive of high cardiovascular risk and silent heart disease. (4) Gouty arthritis has been associated with alteration of the carotid intima media thickness (cIMT) and subclinical atherosclerosis. Cukurova et al studied patients with gout, finding an increase in cIMT compared to patients with asymptomatic hyperuricemia. (5)

Objectives: Assess the carotid intima-media thickness in patients with monosodium urate deposits arthritis 